\title{
Tribological Behavior and Surface Characteristics of Metal Microtube in Flaring Test*
}

\author{
Mohammad Ali MIRZAI** and Ken-ichi MANABE** \\ ** Department of mechanical Engineering, \\ Tokyo Metropolitan University, 1-1 Minami-Osawa, \\ Hachioji-shi, Tokyo, 192-0397 Japan \\ E-mails:mirzaima@center.tmu.ac.jp, manabe@tmu.ac.jp
}

\begin{abstract}
In this study, the effect of tool asperity and the lubrication condition on tribological behavior, inner surface characteristics and deformation limit of metallic microtube in flaring test were investigated experimentally. The microtube used in this experiment is stainless steel (SUS 316L) and has a $500 \mu \mathrm{m}$ outer diameter and $50 \mu \mathrm{m}$ thickness. The flaring tests of the microtube using a conical tool were conducted under dry and two kinds of lubricated-contact conditions as well as different tool surface roughnesses. As a result, it is found that the flaring load and deformation limit of the microtube increase when using a rougher tool. In addition, a spray-type fluorocarbon resin, as a solid lubricant, decreases the above characteristics, but lubrication oil, as a liquid lubricant, exhibits different behavior. Meanwhile, the surface roughnesses of the inner surface of the microtube along axial and circumferential directions reduce when using a rougher tool. From these results, the surface smoothing mechanism of the microtube in the flaring test and the influencing parameters on tribological behavior are discussed.
\end{abstract}

Key words: Mircroforming, Microtube, Flaring Test, Tribology, Deformation Limit

\section{Introduction}

The rising use of microsystems in various applications demands reliable and long-term stable solutions for production problems. In spite of the long history of tribology, there have been many ambiguous and unknown phenomena in tribology systems, particularly in metal forming, up to now. Numerous theoretical and experimental efforts have been made to interpret the tribological behavior and surface characteristics of metallic parts in metal forming processes. For instance, on the macroscale, the smoothing process and the effect of the asperity interactions on the local deformation behavior of a given microcontact were investigated ${ }^{(1)(4)}$, as were the effect of lubricant on the surface characteristics and the open-closed lubricant pockets mechanism ${ }^{(5) \sim(6)}$. In the scaling down of the dimensions of materials and processes from the macroscale to the microscale, some parameters remain constant. The topography of the surface, for instance, is independent of the material dimensions and is one of the most important surface properties. As a result, the ratio between the dimensions of a part and the parameters of the surface roughness changes with miniaturization. This leads to size effects. The tribological behavior apparently changes in the microforming processes, therefore, investigations are necessary in order to clarify the tribological behavior of the micromaterial in metal forming technologies. Although research 
on micro- and nanotribology in different fields are being conducted, there is a very limited amount of investigations on the tribology of microparts in metal forming processes. The deformation behavior of the metal microcomponents is highly dependent on surface interactions. Engel and co-workers ${ }^{(7) \sim(9)}$ extensively investigated the tribological behavior of microparts in metal forming technologies, as well as size effects. They studied microtribology using the double-cup-extrusion (DCE) test. They also suggested a theoretical approach, based on the mechanical-rheological model, to understand and describe the size effect ${ }^{(10) \sim(12)}$. Also, Engel ${ }^{(13)}$ and Engel and Weidel ${ }^{(14)}$ interpreted the tribology in microforming using the model of the open and closed lubricant pockets. On the other hand, Manabe and Nishimura ${ }^{(15) \sim(16)}$ studied the effects of mechanical properties of tubes, the lubricants, and the punch head semicone angle on the flaring process on the macroscale. Furthermore, the stress and strain distribution in conical flaring was also investigated to clarify the deformation behavior. For milli- and microscale, Manabe and co-workers ${ }^{(17) \sim(18)}$ investigated the surface roughnesses of the tool and the blank on deep-drawing processes using an axisymmetric numerical model with the cyclic concave-convex configuration.

Up to now, there have been no reports on the tribology of the metal microtubes in metal forming processes. Since the microtube is one of the most important micromaterials to be used in many microdevices, for instance micropumps, microsensors and microradiators, its tribological behavior is of great interest. Mirzai and co-workers ${ }^{(19)}$ investigated the deformation behavior of the metal microtubes in the flaring test, as well as the flaring limits with different conical tool angles. In addition, by comparison with the macroscale flaring test results, it was found that the behavior of the microtube materials is similar to that of the macrotube materials. The circumferential ductility of a thin-walled tube is evaluated from the value of the flaring limit in the flaring test; on the other hand, the flaring limit is affected by friction and the surface roughness of the tool. Also, because of the size effects as stated above, the effect of the surface roughness becomes more significant in microforming. Therefore, to understand the effect of the tool surface topography and the lubricant conditions on the ductility and the forming limit of the microtube, the study of the tribological behavior and the surface characteristics of the inner surface of the microtube (ISM) in this test will be essential.

The aim of this study is to examine the interfacial surface behavior and the effects of the tool surface topography, as well as the lubricant condition, on the surface characteristics and of the smoothing mechanism on the ISM in the flaring test experimentally. To study the effect of the tool surface topography experimentally, four different surface roughnesses with circumferential asperity pattern were applied to the conical tool, and the flaring tests were performed under various lubricant conditions. Referring to the microscope observation results, we discussed the surface characteristics and deformation behavior on the ISM as well as the effect of the lubricant conditions and the tool asperity in the flaring test for the metal microtube.

\section{Experiment}

\subsection{Material Used}

The metal microtube is made of stainless steel (SUS 316L) and has a $500 \mu \mathrm{m}$ diameter and $50 \mu \mathrm{m}$ thickness. The tensile test was performed to determine the mechanical properties of the microtube, and the results are given in Table 1.

Table 1 Mechanical properties of material used

\begin{tabular}{ll}
\hline Strength coefficient, $\mathrm{k}^{*},(\mathrm{MPa})$ & 447 \\
Strain hardening exponent, $\mathrm{n}^{*}$ & 0.114 \\
Young's modulus, E, $(\mathrm{GPa})$ & 193 \\
Poisson's ratio, $\mathrm{v}$ & 0.3 \\
Total elongation, $\% \quad\left(\mathrm{~L}_{0} \approx 10 \mathrm{~mm}\right)$ & 10.5 \\
\hline$* \sigma=\mathrm{k} \varepsilon^{\mathrm{n}}$ &
\end{tabular}




\subsection{Experimental apparatus and procedure}

Figure 1 shows a schematic illustration of the tube flaring test. A conical tool with the semiangle $\alpha$ is driven into the open end of a tube, with the initial outer diameter $\mathrm{D}_{0}$ and the initial wall thickness $\mathrm{t}_{0}$, that is supported on the opposite end.

In this test, the penetration of the conical tool was stopped upon the appearance of a fracture onset at the final diameter $\mathrm{D}_{\mathrm{f}}$. The tool with a conical angle of $40^{\circ}$ was set up in a computer-controlled universal testing machine with a load capacity of $500 \mathrm{~N}$. The microtube is fixed by the collet chuck and accurately aligned with the conical tool by means of the X-Y table under a digital microscope. Before performing the test, the specimen was cleaned with acetone in an ultrasonic cleaner. Also, before each test, the conical tool and the free end of the fixed microtube were cleaned with acetone to remove any deposits and to ensure a mechanically clean condition. The experiments were conducted under the dry and lubricated conditions at room temperature. The lubricants used were two spray-type lubricants, a fluorocarbon resin and a penetrating oil

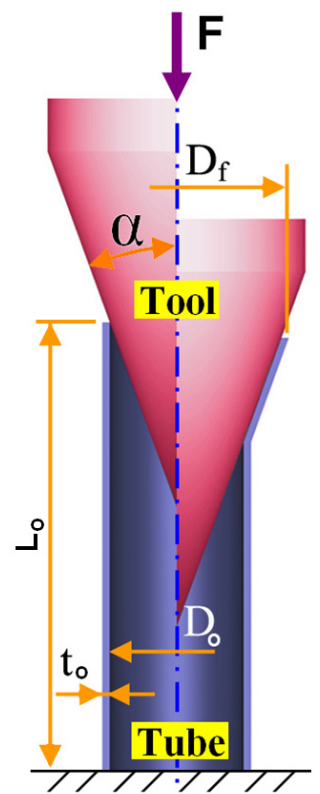

Figure 1 Schematic illustration of tube flaring test containing PTFE and organic molybdenum, which are called in this paper, fluorine and oil, respectively. The fluorine lubricant, five minutes after being sprayed on the tool, changes from liquid to solid, but the oil lubricant is always liquid and its dynamic and kinematic viscosities at $20{ }^{\circ} \mathrm{C}$ are $4 \mathrm{mPa}$.s and $5.26 \times 10^{-6} \mathrm{~m}^{2} / \mathrm{s}$, respectively. The deformation behavior at the edge of the microtube was observed during the test with the digital microscope at a magnification of 150X. Also during the test, the load-stroke curve was followed on a monitor. The tool penetration was stopped when a fracture appeared at the edge of the microtube. Since the tool movement should be stopped immediately after the appearance of a fracture, the minimum speed of the universal testing machine, $0.05 \mathrm{~mm} / \mathrm{min}$, was chosen. After the fracture, the conical tool was removed from the specimen, and then the expansion at the end of the flared zone was measured with the digital microscope.

\subsection{Surface characteristics of original microtube and tools}

Figures 2 and 3 show confocal scanning laser micrographs as well as axial and circumferential roughness profiles of the conical tool and the original microtube in the flaring test. As shown in Fig. 2(a), the ISM is a free surface and very rough because of free tube drawing process. In contrast, the outer surface of the microtube in Fig. 2(b) is smoother than the inner surface because of contact with the drawing die. The surface asperity pattern of the two surfaces has an axial orientation due to the fabrication process. The surface characteristics of the inner surface of the original microtube are given in Table 2. Its average relative roughness deviation to wall thickness is 5 to 10 percentage and markedly higher than the conventional tubes on macroscale.

Three different axial surface roughnesses (arithmetic average roughness $\left(\mathrm{R}_{\mathrm{a}}\right)=0.025$, 0.10 and 0.25 and ten-spot average roughness $\left(\mathrm{R}_{\mathrm{z}}\right)=0.18,0.56$ and $\left.1.95 \mu \mathrm{m}\right)$ were created on the conical tool by grinding the tool against dry emery paper of grit sizes $\# 2000, \# 800$ and $\# 400$ as the tool was rotated in a lathe chuck, and the superfine tool $\left(\mathrm{R}_{\mathrm{a}}=0.005\right.$ and $\left.\mathrm{R}_{\mathrm{z}}=0.02 \mu \mathrm{m}\right)$ was

Table 2 Inner surface roughness of original microtube

\begin{tabular}{c|c|c|c}
\hline \multicolumn{2}{c|}{ Axial } & \multicolumn{2}{c}{$\begin{array}{c}\text { Circumferential } \\
\text { Cut-off }=0.1\end{array}$} \\
\hline $\mathrm{R}_{\mathrm{a}}$ & $\mathrm{R}_{\mathrm{z}}$ & $\mathrm{R}_{\mathrm{a}}$ & $\mathrm{R}_{\mathrm{z}}$ \\
\hline 0.64 & 4.53 & 0.608 & 2.63 \\
\hline
\end{tabular}


made with a centerless grinding machine. From Fig. 3, the surface asperity pattern of the conical tools can be clearly seen that is circumferential owing to the polishing method.
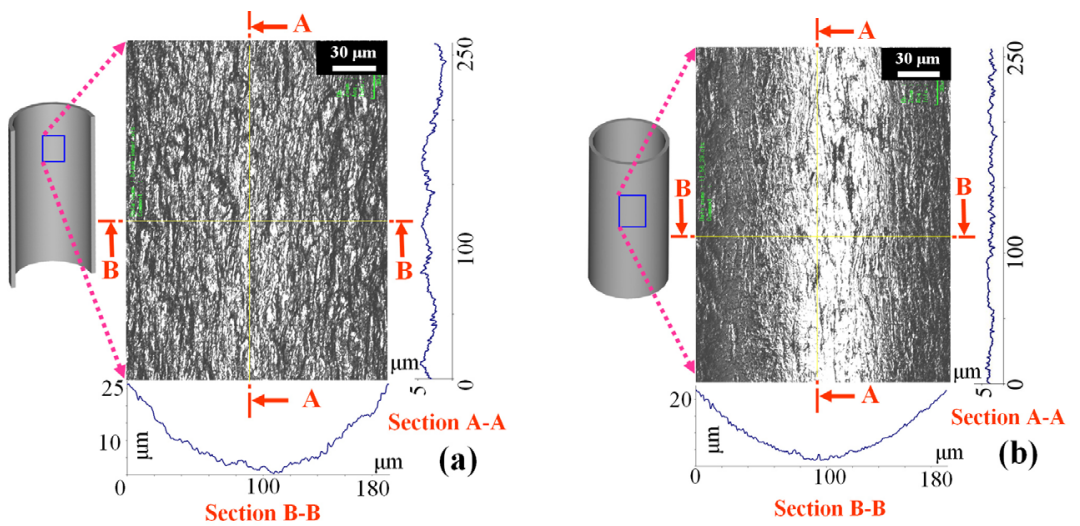

Figure 2 Confocal scanning laser micrographs, as well as axial and circumferential roughness profiles, of blank microtube in flaring test (a) inner and (b) outer surfaces
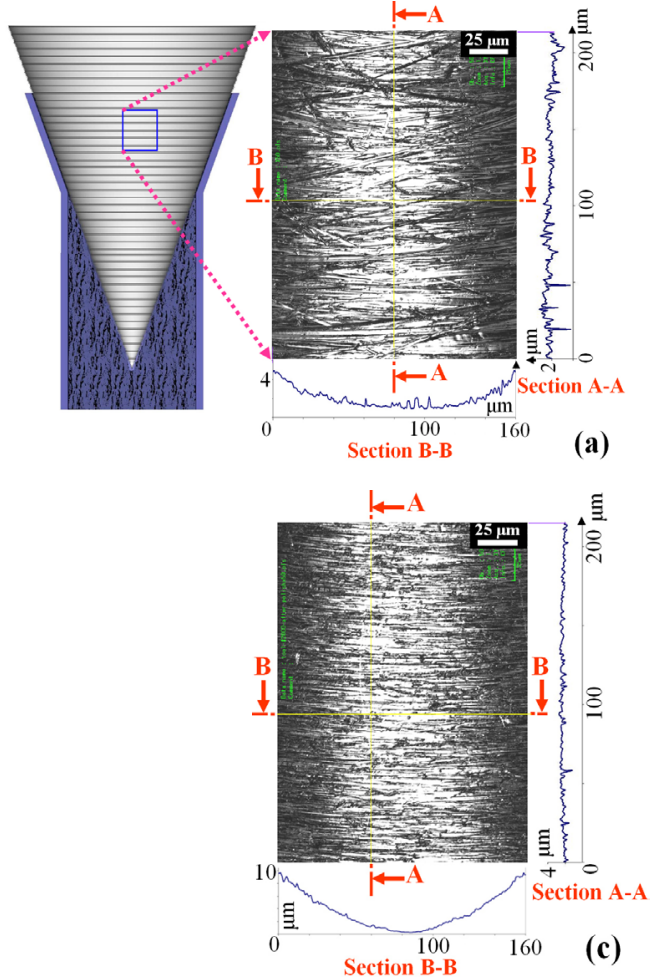
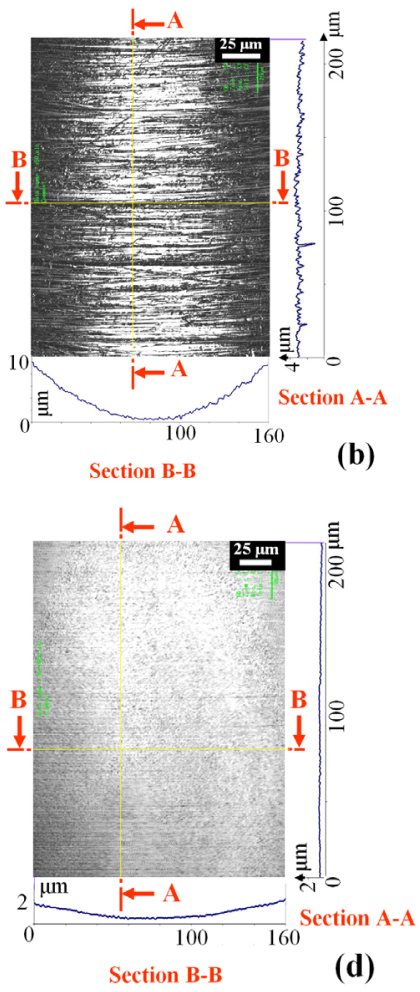

Figure 3 Scanning laser micrographs, as well as axial and circumferential roughness profiles, of conical tool in flaring test: (a) rough conical tool $\left(\mathrm{R}_{\mathrm{a}}=0.25\right.$ and $\left.\mathrm{R}_{\mathrm{z}}=1.95 \mu \mathrm{m}\right)$ (b) medium conical tool $\left(\mathrm{R}_{\mathrm{a}}=0.1\right.$ and $\left.\mathrm{R}_{\mathrm{z}}=0.56 \mu \mathrm{m}\right)$ (c) fine conical tool $\left(\mathrm{R}_{\mathrm{a}}=0.025\right.$ and $\left.\mathrm{R}_{\mathrm{z}}=0.18 \mu \mathrm{m}\right)(\mathrm{d})$ superfine conical tool $\left(\mathrm{R}_{\mathrm{a}}=0.005\right.$ and $\left.\mathrm{R}_{\mathrm{z}}=0.02 \mu \mathrm{m}\right)$

\section{Results and Discussion}

\subsection{Load-stroke curves}

Figure 4 shows load-stroke curves of the microtube in the flaring test with the different lubricants and different the surface roughnesses of the conical tool. The deformation behavior of the microtube does not change with the lubricant condition or the tool asperity applied in this study because the path and the tendency of the curves are similar. However, their quantities, particularly the load peaks of the curves, are affected by the tool surface 
roughness and the lubricant condition. As shown in all graphs in Fig. 4, the load peaks in the case of the fluorine lubricant are minimum. However, in the case of the oil lubrication, the load-stroke curves have different behaviors with different tool surface roughnesses. As shown in Figs. 4(a) and (b), these curves are different from the load-stroke curves for the dry condition using the superfine and the fine tools. However, Figs. 4(c) and (d) show that these curves are very near to the load-stroke curves for the dry condition using the medium and the rough tools.

Figures 4(e), (f) and (g) are rearranged Figs. 4(a) to (d) from a viewpoint of lubricant conditions. These show that the load-stroke curves shift with increasing tool surface roughness. It seems that when the microtube, as softer material, is pressed and slid on the conical tool, which is the harder material, the softer material will deform plastically in axial and circumferential directions. Since the softer material must flow over the asperities of the harder material and the rough tool has more surface asperities, mechanical adhesion and subsequent friction force increase as the tool surface roughness increases. Thus, due to the friction, the flaring load increases with increasing tool surface roughness.
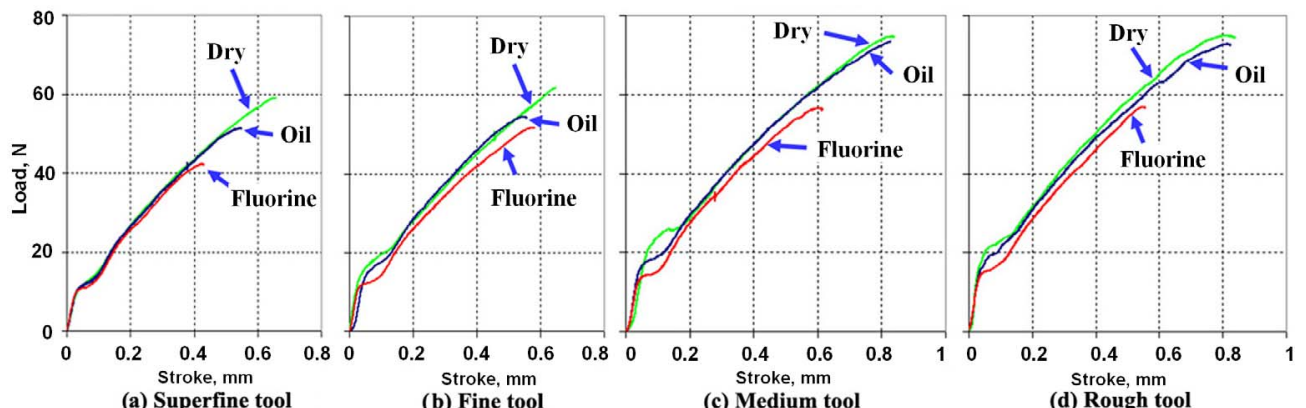

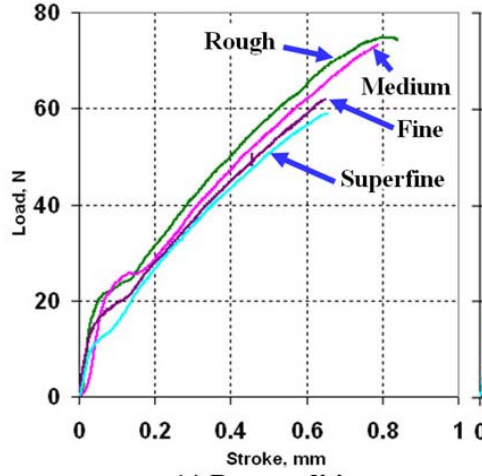

(e) Dry condition

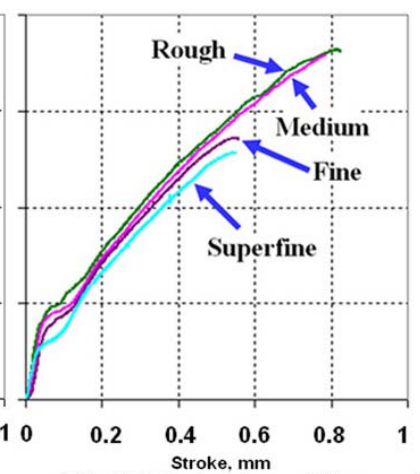

(f) Oil lubricant condition

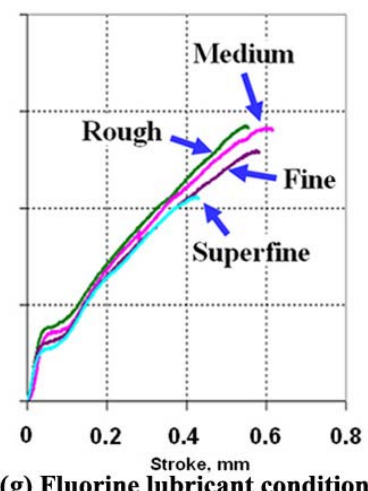

(g) Fluorine lubricant condition

Figure 4 Load-stroke curves obtained from flaring test with different tool surface roughnesses: (a) superfine, (b) fine, (c) medium and (d) rough conical tool, and with (e) dry, (f) oil and (g) fluorine lubricant conditions

\subsection{Microtube surface characteristics after flaring test}

The investigation of the surface characteristics of a specimen in a tribology system is one of the best ways of understanding the nature and consequences of tribological behavior and of solving the technological problems. In this section, the inner surface characteristics of the microtube after flaring test in the case of different lubricant conditions as well as different tool surface roughnesses are examined.

Figure 5 shows the confocal scanning laser micrographs as well as axial and circumferential roughness profiles of the ISM in the flaring test with the foresaid conditions. In these figures, the bright part shows the smoothed ISM, that increases with increasing tool asperity. In addition, in the case of the oil lubricant condition, the bright part is more than that under other lubricant conditions. In contrast, the dark parts show 


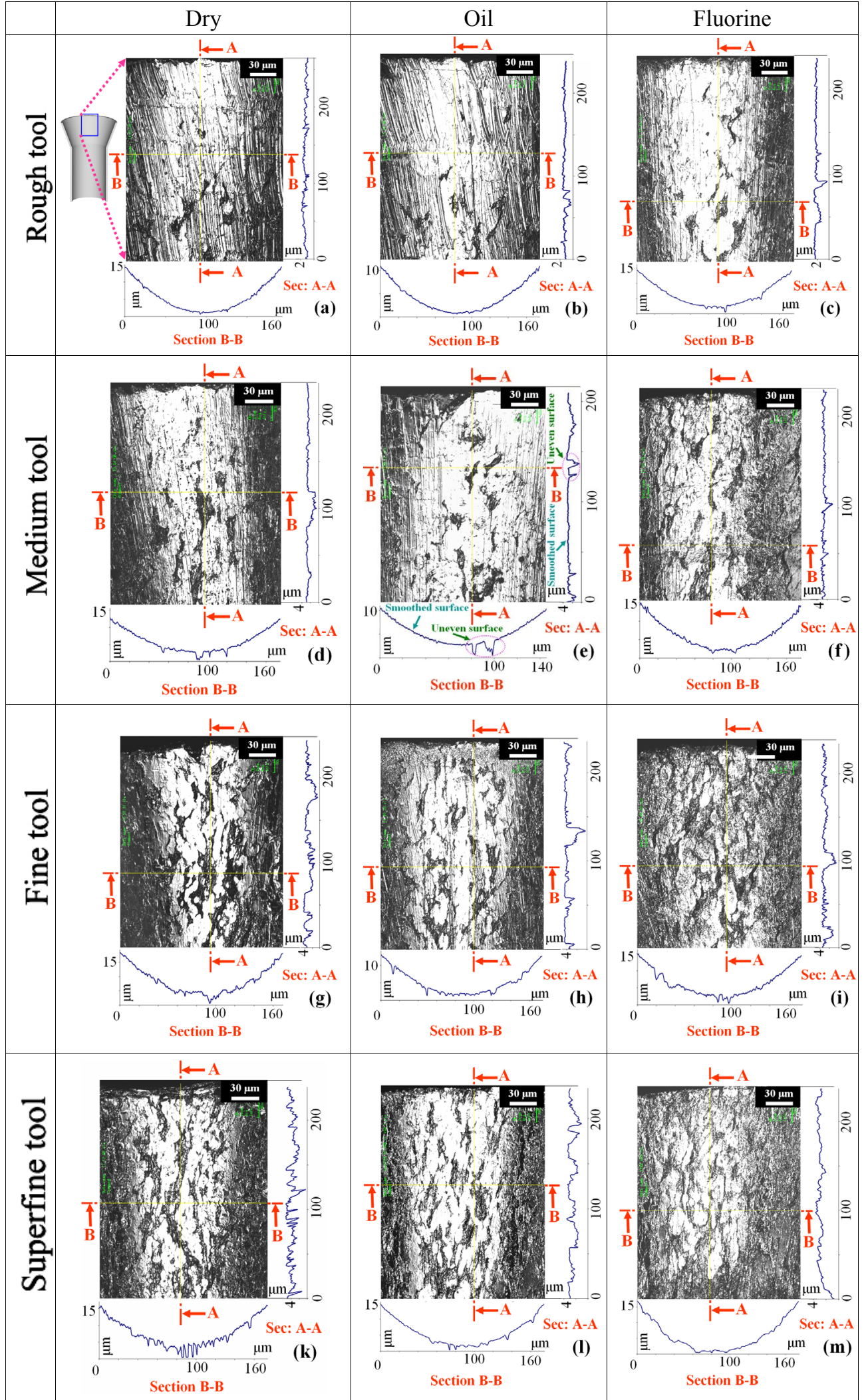

Figure 5 Confocal scanning laser micrographs, as well as axial and circumferential roughness profiles, of the microtube obtained in flaring test using rough tool with (a) dry, (b) oil and (c) fluorine lubricant conditions, and medium tool with (d) dry, (e) oil and (f) fluorine, and fine tool with (g) dry, (h) oil and (i) fluorine, and superfine tool with (k) dry, 
the uneven surfaces that have not been contacted by the conical tool at all; therefore they have not been smoothed. On the other hand, some scratches are seen on the ISM owing to adhesion, particularly mechanical adhesion, and increase as the tool surface roughness increases. The axial and circumferential roughness profiles of the inner surfaces of the microtube obviously indicate combination of the smoothed and the uneven surfaces formed by the flaring test and confirm the above descriptions showing in Fig. 5. The surface roughness characteristics of the ISM after the flaring test are measured with a confocal laser microscope along the axial and circumferential directions.

Table 3 lists the surface characteristics of microtube after the test as the average roughness at 15 positions in every micrograph of several locations for different tool asperity conditions. Since the surface of the microtube in the circumferential direction is located along an arc, a cut-off value of 0.1 is considered in the circumferential direction in order to measure the surface roughness along a straight line.

Figure 6 shows the effects of tool roughness and lubricant conditions on the surface roughness of the ISM after flaring test. From Figs. 6(a) and (b), it is obviously seen that $\mathrm{R}_{\mathrm{a}}$ of the ISM in the flaring test along the axial and the circumferential directions increases with decreasing tool asperity under the dry and the lubricant conditions. Also, $\mathrm{R}_{\mathrm{a}}$ of the ISM in the flaring test with different tool asperities is minimum in the case of the oil lubricant. In contrast, it is maximum under the dry condition.

From Figs. 6(c) and (d), it is found that $\mathrm{R}_{\mathrm{z}}$ of the ISM in the flaring test under the dry condition along the axial and the circumferential directions has a similar tendency to that in Figs. 6(a) and (b). However, it is irregular in the case of the oil and fluorine lubricants. $\mathrm{R}_{\mathrm{a}}$ is the calculated average of all deviations of the roughness profile from the median line over a predefined length. But $\mathrm{R}_{\mathrm{z}}$ is just the mean value of 5 individual roughness depths obtained from consecutive samples of the roughness profile. Therefore $R_{a}$ shows the average surface characteristics of the surfaces better than $R_{z}$ and it is more applicable and important in many reports.

Also, compared between the surface characteristics of the ISM before and after the flaring test in axial and circumferential directions in Fig. 6, it is found that the difference between surface roughness of the ISM in axial direction before and after the test is more than them in circumferential direction. It means that the smoothing process in axial direction is more effective than in circumferential direction because of the surface

Table 3 Surface characteristics of microtube after flaring test

\begin{tabular}{|c|c|c|c|c|c|}
\hline \multirow{3}{*}{$\begin{array}{l}\text { Lubricant } \\
\text { condition }\end{array}$} & \multirow{3}{*}{$\begin{array}{c}\text { Tool } \\
\text { surface } \\
\text { asperity }\end{array}$} & \multicolumn{4}{|c|}{ Inner surface } \\
\hline & & \multicolumn{2}{|c|}{ Axial } & \multicolumn{2}{|c|}{$\begin{array}{c}\text { Circumferential } \\
\text { Cut-off }=0.1\end{array}$} \\
\hline & & $\mathrm{R}_{\mathrm{a}}$ & $\mathrm{R}_{\mathrm{z}}$ & $\mathrm{R}_{\mathrm{a}}$ & $\mathrm{R}_{\mathrm{z}}$ \\
\hline \multirow{4}{*}{ Dry } & Rough & 0.235 & 2.017 & 0.422 & 1.451 \\
\hline & Medium & 0.267 & 2.282 & 0.435 & 1.69 \\
\hline & Fine & 0.473 & 3.463 & 0.528 & 2.297 \\
\hline & Superfine & 0.499 & 4.579 & 0.553 & 2.602 \\
\hline \multirow{4}{*}{ Oil } & Rough & 0.209 & 1.970 & 0.372 & 1.264 \\
\hline & Medium & 0.247 & 2.466 & 0.374 & 1.425 \\
\hline & Fine & 0.300 & 2.921 & 0.495 & 2.181 \\
\hline & Superfine & 0.316 & 2.318 & 0.551 & 1.985 \\
\hline \multirow{4}{*}{ Fluorine } & Rough & 0.215 & 2.459 & 0.385 & 1.211 \\
\hline & Medium & 0.260 & 2.870 & 0.432 & 1.468 \\
\hline & Fine & 0.367 & 2.821 & 0.505 & 1.869 \\
\hline & Superfine & 0.370 & 2.983 & 0.514 & 1.385 \\
\hline
\end{tabular}


topography of the conical tool and direction of tool movement in this test. Also in Figs. 6(a) and (b), the $\mathrm{R}_{\mathrm{a}}$ of the ISM after the flaring test in the both directions is less than that before the test for all different conditions. On the other hand, the $\mathrm{R}_{\mathrm{z}}$ of the ISM after the test in the both directions is very close to that before the test for some cases. This is caused by different methods of calculation for the $\mathrm{R}_{\mathrm{a}}$ and $\mathrm{R}_{\mathrm{z}}$ described above.

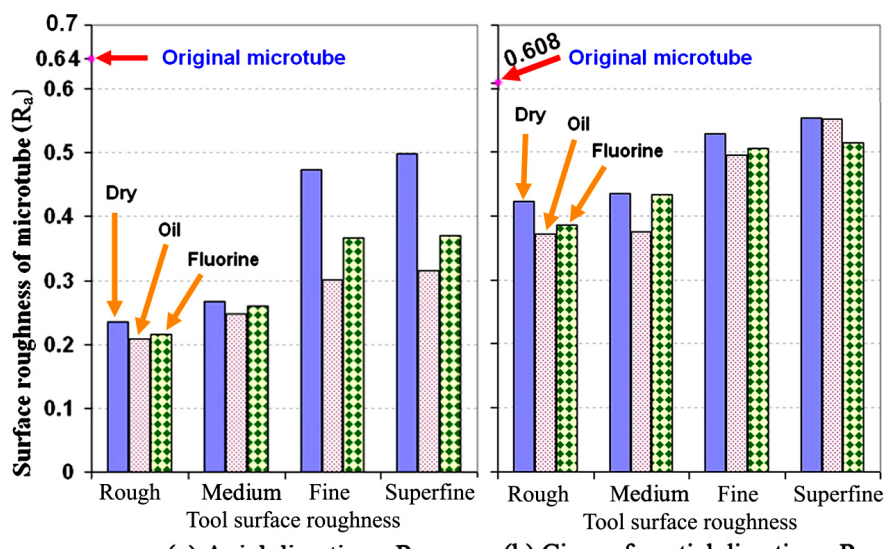

(a) Axial direction - $\mathrm{R}$

(b) Circumferential direction $-\mathrm{R}_{\mathrm{a}}$

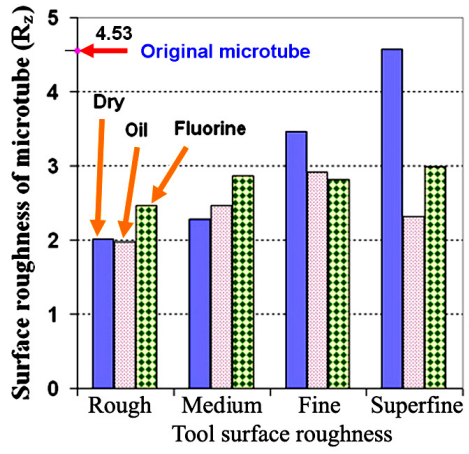

(c) Axial direction $-\mathrm{R}_{\mathrm{z}}$

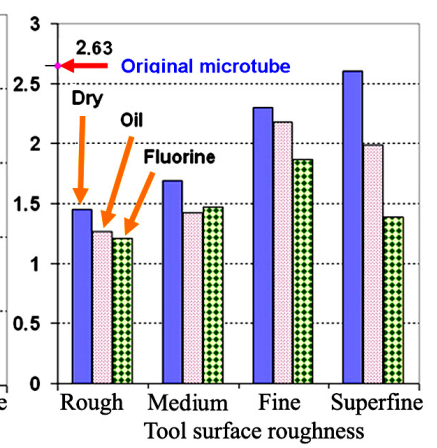

(d) Circumferential direction $-\mathbf{R}_{z}$

Figure 6 Roughness of inner surface of microtube after flaring test as arithmetic average roughness $\left(\mathrm{R}_{\mathrm{a}}\right)$ (a) in axial and (b) circumferential directions, and as average roughness depth $\left(R_{z}\right)(c)$ in axial and (d) circumferential directions

\subsection{Smoothing mechanisms of microtube in flaring test}

As described in the previous section, the tool asperity and the lubricant conditions affect the surface characteristics of the ISM. Mirzai and Manabe ${ }^{(20)}$ studied the effect of the tool surface topography on the interfacial deformation behavior of the microtube and the smoothing mechanism in the flaring test by the numerical and experimental methods. In their paper, the most of the smoothing of the ISM in the flaring test was revealed to occur by a compression-sliding mechanism when using the fine tool and by an interlocking mechanism when using the rough tool. The contributions of the interlocking and compression-sliding mechanisms in the smoothing process of the ISM are suggested to change owing to combination asperities of the conical tool and the ISM. Since the ISM is rough, the interlocking mechanism is more effective than the compression-sliding mechanism in the smoothing process of the ISM in the flaring test with the rough tool. Thus the asperity of the ISM decreases when using a rougher tool.

On the other hand, as described in the previous section, the surface characteristics of the ISM are affected by the lubricant used. According to the results shown in Figs. 5 and 6 , the surface characteristics of the ISM after the flaring test with the oil and fluorine lubrications are close to those obtained under the dry condition. Therefore, it can be understood from these results that the smoothing process of the ISM under the oil and 
fluorine lubrications is similar to that under the dry condition.

Figure 7 shows the schematic illustration of smoothing mechanisms on the ISM in the flaring test using the rough and fine tools under the oil lubrication condition. When using a rough tool, it is supposed that the oil escapes from between the ISM and the rough tool because of the big gap between them as well as the axial roughness orientation on the ISM. Therefore the oil could not be trapped within them and open lubricant pockets (OLPs) are generated. In this case, the most of smoothing process on the ISM is done by interlocking mechanism. Because, as shown in Fig. 8(a), the asperities on the ISM are not uniform and the ISM has many hills and valleys although the roughnesses orientation on the ISM is macroscopically axial. Thus they are able to engage with the asperities of the rough tool which have circumferential orientation. When using a fine tool, the oil is trapped within the ISM and the tool surface because of small gap between them as well as the smooth surface of the fine tool. Therefore closed lubricant pockets (CLPs) are generated. In this case, the most of smoothing process on the ISM, in less dependence on roughness orientation of interfacial surfaces, is done by compression-sliding mechanism. Because the asperities on the fine tool, in contrast the asperities on the ISM, are small.

In Fig. 7(b), the distribution of pressure on the rough and fine tools at the fifth step of schematic smoothing process of the ISM shown in Fig. 7(a) is indicated. A convex surface of rough tool can push on the ISM at direct contact positions characterized by the pressure $\mathrm{P}_{\mathrm{r}}$ because of the presence of OLPs. Meanwhile the fine tool can push on the ISM at direct contact positions and create the presence of CLPs, and then the pressurized oil lubricant is trapped in the CLPs, which characterized by the pressures $\mathrm{P}_{\mathrm{f}}$ and $\mathrm{P}_{\mathrm{l}}$, respectively.

Figure 8 shows SEM micrographs of the smoothed ISM after the flaring test with the rough and fine tools. These micrographs provide an experimental confirmation on the validity of the effect described above. In Fig. 8(b), many scratches are clearly observed on the smoothed ISM, suggesting that it became rough owing to mechanical adhesion between the rough conical tool and the ISM. On the contrary, in Fig. 8(c), the smoothed ISM is shown to be fine, and in addition, the CLPs are clearly observed on the ISM. Since the CLPs and the OLPs affect friction resistance, it is hypothesized that they affect the load-stroke curve as well as the forming limit. These subjects are discussed in the next section.

As described ${ }^{(20)}$, the surface roughness of the ISM decreased when using a rougher tool because of the above described smoothing mechanisms under the various combinations of the asperities of the tool and the microtube. Also, since adhesion is possible to occur easily under the dry condition, and not for the oil and the fluorine lubricant conditions, the surface roughness of the ISM increases in case of the dry condition. On the other hand, when the fluorine lubricant is used as solid lubricant, it fills in the surface asperities and the gaps between the ISM and the conical tool, and creates a smooth and slippery coating layer. Thus, the solid lubricant is more difficult to escape from the gap between the microtube and
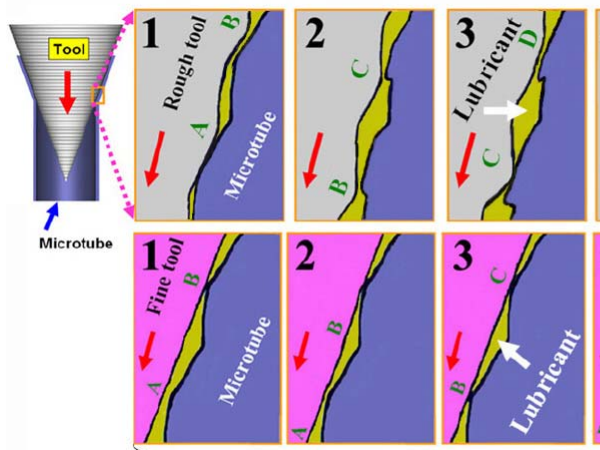

(a)
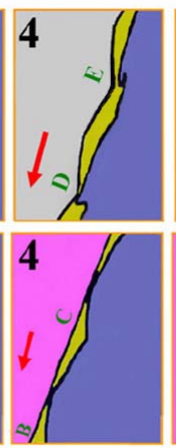
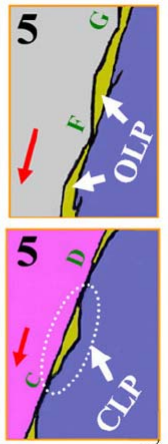

Figure 7 Schematic representation of (a) smoothing process on ISM in flaring test using rough and fine tools with oil lubricant (b) distribution of pressure on rough and fine tools 

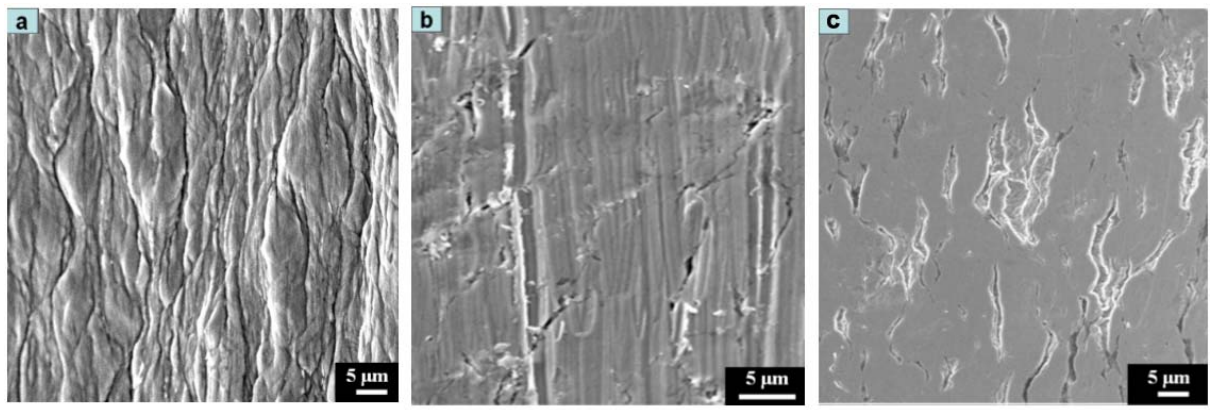

Figure 8 SEM micrographs of inner surface of microtube (a) before and (b) after flaring test when using rough and (c) fine tools

the tool, and makes less direct contacts between their surfaces so that is less effective for smoothing on the ISM. In contrast, in the case of the oil lubrication with low viscosity, it easily escapes from the gap between the microtube and the tool. From the results, it is concluded that the ISM in the flaring test with the fluorine lubricant becomes rougher than that in the case of the oil lubricant. In other words, the smoothed ISM in the flaring test with the oil lubricant is higher than that with the fluorine lubricant. The experimental results shown in Fig. 5 can be explained by this mechanism.

We note that the smoothing mechanisms on the ISM in the flaring test with the rough and fine tools and fluorine lubricant are similar to the mechanisms shown in Fig. 7.

\subsection{Flaring limit of microtube}

Two instability phenomena dominate the expansion limit of the tube in the flaring test. The first one is buckling of the straight part of the tube. In this study, the suitable microtube length of $2 \mathrm{~mm}$ was chosen to prevent the buckling instability, which was calculated by theoretical and numerical methods.

The second instability is "necking", local thinning and then fracture takes place at the conical edge of the tube. In fact, this instability governs the circumferential ductility, which represents the flaring limit of the microtube. The flaring limit is related to the circumferential ductility of the microtube and is determined as a maximum flared expansion. In this study, the flaring limit, $\lambda_{\mathrm{f}}$ is defined as

$$
\lambda_{f}=\frac{D_{f}}{D_{0}}-1
$$

where $D_{0}$ and $D_{f}$, as shown in Fig. 1, are the initial and the final maximum expansion diameters of the microtubes, respectively.

Figure 9 shows the variation in flaring limit $\lambda_{\mathrm{f}}$ of the microtube with tool surface roughness showing effect of lubrication condition. From this figure, it can be seen that the flaring limit $\lambda_{\mathrm{f}}$ increases with increasing tool asperity.

As described in previous sections, friction resistance increases with the increase in tool asperity. On the other hand, fracture is delayed in the flaring test with increasing friction. This means the flaring limit increases with increasing surface roughness of the conical tool.

Regarding the effect of lubricant condition, the fluorine lubrication causes lower flaring limit and there is less tool asperity dependence.

In the case of oil lubrication, the flaring limit is dependent on the tool asperity. The limit for oil lubrication is close to that under dry condition with the rough $\left(R_{\mathrm{a}}=0.25 \mu \mathrm{m}\right)$ and the medium $\left(R_{\mathrm{a}}=0.1 \mu \mathrm{m}\right)$ tool, and be that under the fluorine lubricant condition with the fine $\left(R_{\mathrm{a}}=0.025 \mu \mathrm{m}\right)$ and superfine $\left(R_{\mathrm{a}}=0.005 \mu \mathrm{m}\right)$ tools. Their tendency coincides with the behavior of the load-stroke curves in Figs. 4(a), (b), (c) and (d). Since the CLPs are created using the fine and the superfine tools as described in the previous section, they play 


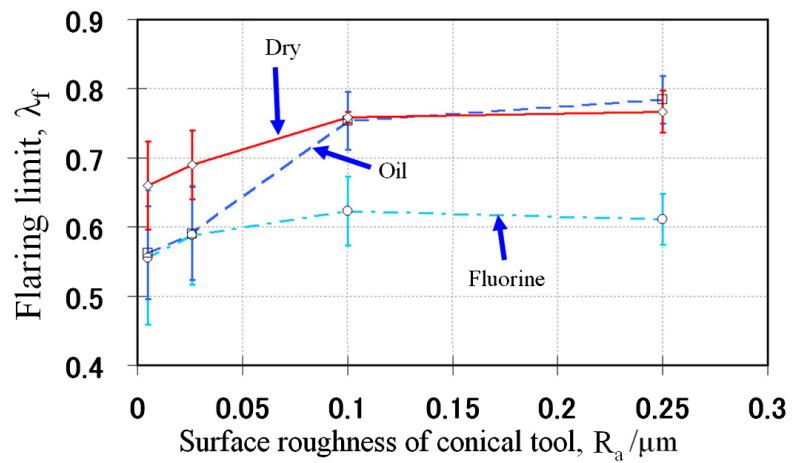

Figure 9 Effect of tool surface roughness and lubrication condition on flaring limit

a role of friction reduction. As a result, the flaring limit as well as the deformation load decreases. In contrast, the OLPs are created when using the rough and medium tools. Lubrication condition similar to OLPs can be made under the fluid oil lubrication with low viscosity, which can easily escape from the gap between the tool and the microtube. In other words, this is similar condition to dry condition so that the friction becomes high and consequently both the deformation load and the flaring limit increase and become close to those under the dry condition.

In general, the microtube has greater relative roughness variation to wall thickness compared to the conventional macrotube, so that surface topography of the microtube and the tool significantly affects triborogical behavior and deformation characteristics of microtube in the flaring test.

\section{Conclusions}

The conclusions based on the experimental results are as follows:

(1) The flaring load in the load-stroke curves as well as the deformation limit in the flaring test for a microtube increases with increasing tool surface roughness because of mechanical adhesion. Compared with the dry condition, they decrease when fluorine is used as solid lubricant. Also, because of the open lubricant pockets, they do not significantly change in the case of lubrication oil with the rough and medium tools. In contrast, because of the presence of the closed lubricant pockets, they decrease in cases of the fine and the superfine tool with the lubrication oil.

(2) The surface roughness of the inner surface of the microtube, owing to different smoothing mechanisms, decreases with increasing tool surface asperity. Because of the increase in the mechanical adhesion and subsequent friction with increasing tool surface asperity, the scratches on the ISM increase when using a rougher tool. Therefore its surface roughness increases in the case of the dry condition. Also in the case of fluorine lubricant (solid lubricant), the ISM after the flaring test becomes rougher than that under the oil lubricant condition because that a solid lubricant is more difficult to escape from the gap between the microtube and the tool, and makes less direct contact condition between them.

(3) For microtubes, the open and closed lubricant pockets strongly affect not only friction and subsequent flaring load and deformation limit but also the tribological characteristics interacting with the tool and microtube surfaces and the viscosity of lubricant.

\section{Acknowledgments}

This project was supported by JSPS and RFBR under the Japan-Russia Research Cooperative Program. The authors would like to express their thanks to Professor S. Kataoka from Shonan Institute of Technology for his valuable advice. The first author would like to acknowledge the Ministry of Science, Research and Technology of Iran and Hormozgan University for their financial support. 


\section{References}

(1) Lo, S., Yang, T., A new mechanism of asperity flattening in sliding contact-the role of tool elastic microwedge, ASME Journal of Tribology, Vol. 125, (2003), pp. 713-719.

(2) Lo, S., Yang, T., A microwedge model of sliding contact in boundary/mixed lubrication, Wear, Vol. 261, (2006), pp. 1163-1173.

(3) Chang, W. R. et al., An elastic-plastic model for the contact of rough surfaces, ASME Journal of Tribology, Vol. 109, (1987), pp. 257-263.

(4) Wang, Z., et al., FEM simulation of surface smoothing in the ironing process, Journal of Materials Processing Technology, Vol. 113, (2001), pp. 705-709.

(5) Azushima, A., Tanaka, T., Lubricant behavior trapped within pockets on workpiece surface in lubricated upsetting by means of direct fluorescence observation technique, CIRP Annals-Manufacturing Technology, Vol. 49, (2000), pp. 165-168.

(6) Lo, S., Tsai, S., Real-time observation of the evolution of contact area under boundary lubrication in sliding contact, ASME Journal of Tribology, Vol. 124, (2002), pp. 229-238.

(7) Engel, U., Eckstein, R., Microforming - from basic research to its realization, Journal of Materials Processing Technology, Vol. 125-126, (2002), pp. 35-44.

(8) Geiger, M., Engel, U., Microforming - a challenge to the plasticity research community, Journal of the Japan Society for Technology of Plasticity, Vol. 43, (2002), pp. 171-172.

(9) Engel, U. et al., Cold forging of microparts - effect of miniaturization on friction, $1 s t$ International ESAFORM Conference on Material Forming, France, (1998), pp. 77-80.

(10) Geißdörfer, S. et al., FE-simulation of microforming processes applying a mesoscopic model, International Journal of Machine Tools and Manufacture, Vol. 46, (2006), pp. 1222-1226.

(11) Engel, U. et al., Simulation of microforming processes - An advanced approach applying a mesoscopic model, 8th International Conference on Technology of Plasticity, Vol. 173, Italy, (2005), pp. 447-448.

(12) Geißdörfer, S., Engel, U., Mesoscopic model-simulation of size effects in microforming, 10th International Conference on Metal forming, Poland, (2004), pp. 699-703.

(13) Engel, U., Tribology in microforming, Wear, Vol. 260, (2005), pp. 265-273.

(14) Engel, U., Weidel, S., Evaluation of surface topography in microforming, 3rd International Conference on Tribology of Manufacturing Processes, Japan, (2007), on CD.

(15) Manabe, K., Nishimura, H., Forming loads in tube-flaring with conical punch - study on nosing and flaring of tubes V, Journal of the Japan Society for Technology of Plasticity, Vol. 24, (1983), pp. 47-51.

(16) Manabe, K., Nishimura, H., Stress and strain distributions in tube-flaring with conical punch - study on nosing and flaring of tubes VI, Journal of the Japan Society for Technology of Plasticity, Vol. 24, (1983), pp. 276-282.

(17) Manabe, K. et al., Evaluation of milli-scale cylindrical cup in two-stage deep drawing process, Journal of Materials Processing Technology, Vol. 187-188, (2007), pp. 245-249.

(18) Manabe, K. et al., Validation of FE simulation based on surface roughness model in micro-deep drawing process, Journal of Materials Processing Technology, Vol. 204, (2008), pp. 89-93.

(19) Mirzai, M. A. et al., Deformation characteristics of microtubes in flaring test, Journal of Materials Processing Technology, Vol. 201, (2008), pp. 214-219.

(20) Mirzai, M. A., Manabe, K., Effect of tool surface topography on interfacial deformation behavior of metal microtube in flaring test, Journal of Solid Mechanics and Materials Engineering, (2008), submitted. 